\title{
Spin labeling ESR investigation of the molecular environment of soil interacting with chemical organic contaminants
}

\author{
Olga N. Aleksandrova* \\ Ural Federal University, Mira Street 19, Ekaterinburg, Russia \\ University of Osnabrueck, Institute of Environmental Systems Research (USF), Barbarastrasse 12, 49076 Osnabrueck, Germany
}

\section{A R T I C L E I N F O}

Article history:

Received 8 June 2012

Accepted 17 December 2012

Available online 9 January 2013

\section{Keywords:}

Electron spin resonance analysis

Spin labeling

Nitroxide radicals

Organic xenobiotic chemicals

Humic substances

Organic radicals

\begin{abstract}
A B S T R A C T
This manuscript elucidates the influence of special functional groups of organic xenobiotic chemicals on partly simultaneous molecular binding mechanisms. Organic xenobiotics are released as contaminants into the environment and are partially bound to soil constituents, such as humic substances (HS) and the mineral phase. The interaction of chemical contaminants with HS of soil was investigated using a labeling technique of soil samples with stable nitroxide radicals as model contaminants. These nitroxide radicals only differed in one functionality, a hydroxy or an amino functional group. Electron spin resonance (ESR) analyses of contaminated soil samples showed that the interaction was a multistage process that comprised an initial increase in the concentration of free organic radicals of soil influenced by contaminants with hydroxy or amino functionality, a decrease in the polarity of the molecular environment of soil, and a change in mobility of contaminant molecules. Towards the end of interaction, the concentration of free organic radicals usually decreased. Binding of contaminants to soil constituents via specific functionality was revealed detecting a change in their ESR spectra. Basing on the ESR analyses, a two-way exchange of protons' electron pairs between contaminants and HS was hypothesized to interpret the beginning of interaction.
\end{abstract}

c) 2013 Elsevier B.V. All rights reserved.

\section{Introduction}

The protective function of humic substances (HS) ${ }^{1}$ in soils is well known (Aleksandrova et al., 2008, 2010; Eckart, 2001; Ferenc, 1994; Nowak et al., 2011; Perminova and Hartfield, 2005; Perminova et al., 2006). In polluted soils as a result of the diverse reactivity, HS can interact with all classes of ecotoxicants forming stable complexes with contaminants, such as heavy metals, radionuclides, pesticides, biocides, veterinary pharmaceuticals, etc. (Bollag and Myers, 1992; Junge et al., 2011; Nowak et al., 2011; Perminova and Hartfield, 2005; Senesi, 1992). HS produce adducts and charge transfer complexes with hydrophobic organic compounds as well as mediate redox reactions of transition metals, chlorinated and nitrated hydrocarbons (Perminova and Hartfield, 2005). HS can form and promote the formation of non-extractable residuals (NER), ${ }^{1}$ by binding organic chemical xenobiotics or products of their disintegration, thus in turn immobilizing contaminants (Cruz et al., 2011; Matthies et al., 2008; Nowak et al., 2011). However, this immobilization only leads to partial detoxification of contaminants. A large variety of recent works

\footnotetext{
* Tel.: +7 343 3759718, + 495419693438 ; fax: + 495419692599.

E-mail addresses: aleks_olga_upi@inbox.ru, Olga.Aleksandrova@uni-osnabrueck.de.

1 Abbreviations: ESR, electron spin resonance; HS, humic substances; NER, non-extractable residual; OR, organic radicals; SL, spin label; NR, nitroxide radicals; $\mathrm{HQ}$, polymerized hydroquinone; ME, soil from the site Merzenhausen; KA, soil from the site Kaldenkirchen.
}

has shown that contaminants, bound to soil constituents in environmentally relevant concentrations, exert selective pressure on soil microorganisms (Schauss et al., 2009; Zarfl et al., 2009). The estimation of adverse effects of the accumulated NER of contaminants requires improved understanding of molecular mechanisms of the interactions between contaminants and soil constituents. Numerous studies on NER have enabled precise quantitative analyses. However, there is still no information on the chemical structure of NER from tested compounds (Cruz et al., 2011; Gulkowska et al., 2012; Nowak et al., 2011). The role of organic radicals (OR ${ }^{1}$ in binding of contaminants to soil constituents remains under discussion. The complexity of this problem deals with peculiarities of HS that have non-stoichiometric elemental compositions, irregular structures, heterogeneous structural units, and polydisperse molecular weights.

The binding processes of chemical contaminants to HS have already been investigated, using SL and ESR analyses. However, these studies were mostly concerned with the investigations of hydrophobic interactions between SL and humic acids, as well as with the accumulation of hydrophobic SL by the hydrophobic interior of HS. In this study, ESR analysis was found to be a powerful tool to analyze the process, in which organic radicals can be observed and measured as intermediates. (Chien et al., 1997; Ferreira et al., 2001; Paul et al., 2006; Spagnuolo et al., 2005). In addition, the advantage of the ESR approach was revealed by investigations of samples taken from natural soils, because complex processes in soil are substantially connected with free OR and require numerous specific 
measurements to receive valid results (Aleksandrova et al., 2011a; Cruz et al., 2011; Skrzypczak-Bonduelle et al., 2008). Based on the method of SL and ESR analysis, the manuscript presented here considers the role of specific functionality of contaminants on their interaction with HS in soil.

The goal of this investigation was to develop an approach of the influence of specific functionalities of chemical contaminant molecules on changes in the molecular environment of soil and their binding mechanism to soil constituents. This study developed a method to label, contaminate and probe the environmental vicinity of molecules of soil constituents with nitroxide radicals (NR) different only in one functional group (Aleksandrova et al., 2011a). The labeled soil was investigated, using electron spin resonance (ESR) analysis. The hyperfine spectrum of NR was very sensitive to any change in their molecular environment and to any restriction of their mobility, such as binding to soil constituents via the special functional group. The ESR signal from OR of soil was found to be in the same magnetic field range as was NR. The advanced technique allows for investigating the interaction between soil constituents and nitroxide molecules with special functionality as a dynamic process (Berliner, 1979, 1998; Buchachenko and Wasserman, 1973; Kuznetsov, 1976; Likhtenshtein, 1976; Lucarini and Mezzina, 2011; Stoll, 2011; Voinov and Smirnov, 2011). The elucidation of the influence of specific functionalities of contaminants on their interaction with soil constituents including HS and mineral components is a key step to understand the complex molecular binding mechanisms of contaminants to soil.

\section{Material and methods}

\subsection{Soil samples}

Soils from two locations in Germany (a cambisol from "Kaldenkirchen field site" (KA) and a luvisol from "Merzenhausen field site" (ME)) were investigated. Soil KA belonged to the profile Kaldenkirchen - Huelst, field name "Am Huesgesweg" (German classification). Soil ME belonged to the profile Merzenhausen, field name "Im Bruehl" (German classification) (Förster et al., 2009; Junge et al., 2011). From both soils, samples were taken from Horizon $A_{p}$. Samples of soil KA were of brown-black color, slightly humified $(1.7 \%)$, somewhat acidified $\left(\mathrm{pH}\left(\mathrm{CaCl}_{2}\right)=\right.$ 5.52 ), and consist of $75.2 \%$ sand, $21.6 \%$ silt, and $3.2 \%$ clay. The content of iron in samples of soil KA was sufficiently high and amounted to $6856 \mathrm{mg} \cdot \mathrm{kg}^{-1}$. Samples of soil ME were of dark brown, slightly humified $(2.1 \%)$, and had a highly dispersed and crumbly structure of sandy loam (6.4\% sand, $78.2 \%$ silt, and $15.4 \%$ clay). The content of iron of soil samples from ME amounted to $380 \mathrm{mg} \cdot \mathrm{kg}^{-1}$. The $\mathrm{pH}$ value of soil samples from $\mathrm{ME}$ was $\mathrm{pH}(\mathrm{KCl})=7.2$. Soil $\mathrm{KA}$ was subjected to wet sieving for $1 \mathrm{~h}$ to obtain a coarse-grained fraction (with diameter of particle $\mathrm{d}>0.63 \mathrm{~mm}$ ) with a small quantity of HS.

\subsection{Preparation of soil samples for ESR measurements}

Prior to chemical analyses, soil samples were dried at the air for some days to remote water. The experimental investigation of soil OR was carried out, using 1-mm glass capillaries for the X-band. Capillaries were filled with the soil samples. Moistening of the soil sample was accomplished directly in the capillary, applying centrifugation. During labeling of soil samples with nitroxide radicals, water added to soil samples already contained paramagnetic substance at a required concentration of $0.3 \mathrm{mM}$. For several days, soil samples labeled with NR were measured by means of ESR spectroscopy.

To characterize the ESR spectrum of soil organic radicals, polymerized hydroquinones (HQ) (Fig. 1) and humic acid (leonardite), obtained from the chemical department of the Moscow State University (Russia), were used (Bolkova, 2007).

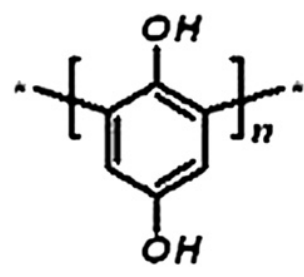

Fig. 1. Polymerized hydroquinones.

\subsection{ESR spectroscopy at $X$-band}

ESR spectra of soil samples were recorded at X-band frequency $(9.43 \mathrm{GHz})$ at room temperature using a spectrometer of the type Magnettech Mini Scope MS 200 (Magnettech GmbH, Analysenmesstechnik, Louis-Blériot-Str. 5, Berlin, Germany) at a microwave power of $10 \mathrm{~mW}$ that corresponds to a microwave attenuation of $10 \mathrm{~dB}$ and a modulation amplitude of $150 \mu \mathrm{T}$. For determination of $\mathrm{g}$ value of soil samples and its change during experiments, DPPH (2,2diphenyl-1-picrylhydrazyl, $g=2.0038$ ) was added to soil and measured simultaneously with soil samples. $100 \mu \mathrm{M}$ solution of stable paramagnetic spin label MTSL (S-(2,2,5,5-tetramethyl-2,5-dihydro-1H-pyrrol3-yl)methyl methanesulfonothioate) was used to determine spin concentrations in soil samples. The chemical compound MTSL was put into a capillary and measured simultaneously with the respective soil sample, which was placed in a second capillary. To correct an influence of water on the measured value of the spin concentration in soil, a second measurement of the same soil sample was carried out simultaneously with the measurement of a pure water sample instead of the sample of MTSL. All spectra were obtained at a receiver gain equal to 100 . To measure and observe the rapid processes expected to take place in the soil sample containing the paramagnetic substances, sweep time and sweep were set to $25 \mathrm{~s}$ and $6.775 \mathrm{mT}$, respectively.

The spectroscopic parameters were quantified using the peak-topeak line width $\mathrm{dB}_{\mathrm{pp}}$, the peak-to-peak height of the derivative signal $\mathrm{I}_{\mathrm{pp}}$ and the approximation $\mathrm{I}_{\mathrm{pp}} \times \mathrm{dB}_{\mathrm{pp}}^{2}$ for the content of $\mathrm{OR}$ in the soil sample.

\subsection{Paramagnetic labels and probes}

Spin labels represent chemically stable paramagnetic molecules to be used as the molecular probes for testing structural properties and molecular mobility of different physical, chemical and biological systems (Tikhonov, 1998). The paramagnetic SL utilized in experiments were NR Tempo (2,2,6,6-Tetramethylpiperidinooxy, Fig. 2a), 4-Amino Tempo (4-amino-2,2,6,6-tetramethyl-1-piperidinylox, Fig. 2b) and 4-Hydroxy Tempo (1-Piperidinyloxy, 4-hydroxy-2,2,6,6-tetramethyl, Fig. 2c), obtained from Aldrich. 4-Amino Tempo and 4-Hydroxy Tempo differed from Tempo in a substituent at the para-position of the piperidine ring. This substituent consists of a typical functional group of organic xenobiotic chemicals, such as of amino or hydroxy functionality, which are susceptible to interaction with HS. In the past, application of hydrophobic paramagnetic substance, such as Tempo and a nitroxide derivative of stearic acid 5-SASL has been studied (Chien et al., 1997; Ferreira et al., 2001). Hydrophobic 5-SASL is well and rapidly sorbed by the hydrophobic interior of the investigated environment of soil. For our purpose to investigate an influence of specific functionality of chemical contaminants on their binding to soil, only nitroxide Tempo seems to be used. Results of the interaction of SL Tempo with soil were used as reference results to compare them with experimental results of the interaction of 4-Amino Tempo and 4-Hydroxy Tempo with soil. Its n-octanol-water partition coefficient $\log \mathrm{K}_{\mathrm{OW}}$ amounts to 1.85 . The partition coefficient of 4-Amino Tempo and 4-Hydroxy Tempo amounts to -1.4 and 0.63 , respectively (Fuchs et al., 1989). 
A<smiles>CC1(C)CCCC(C)(C)N1[O]</smiles>

B<smiles>CC1(C)CC(N)CC(C)(C)N1[O]</smiles>

$\mathrm{C}$<smiles>CC1(C)CC(O)CC(C)(C)N1O</smiles>

Fig. 2. (A) - Chemical structure of nitroxide spin label Tempo used at the experiment. (B) - Chemical structure of nitroxide spin label 4-AminoTempo used at the experiment. (C) - Chemical structure of nitroxide spin label 4-HydroxyTempo used at the experiment.

\section{Results}

\subsection{Investigation of ESR spectra of soil OR}

Soils contain radicals in high abundance. The ESR spectrum of soil is shown in Fig. 3 (left). The ESR signals of OR of soils ME and KA are shown in Fig. 3 (right). The main spectral line corresponding to OR in soils is characterized by g-factor of 2.003951 in soil KA and 2.003967 in soil ME, respectively. The spectrum of soil OR was substantially overlapped with the ESR signal from paramagnetic defects of the mineral phase. In Fig. 3 (right), the right part of the spectral signal was induced by mineral paramagnetic centers $E^{\prime}$ (SkrzypczakBonduelle et al., 2008). In addition, paramagnetic molecules including molecular oxygen that diffuse into the mineral matrix, substantially influenced the shape of the ESR spectrum of soil OR. They broadened the spectral line of soil OR.

The amplitude of the OR signal of soil ME was about three times higher than the amplitude of the OR signal of soil KA, whereas the line width of both soils was approximately $0.36 \mathrm{mT}$. Different signal amplitudes point at different concentrations of free OR in the two soils. The concentration of free $\mathrm{OR}$ in soil $\mathrm{ME}$ amounted to

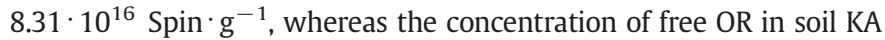
amounted to $2.95 \cdot 10^{16} \mathrm{Spin} \cdot \mathrm{g}^{-1}$. In Fig. 3 (right), ESR spectra of both soils were compared with the ESR signal of the coarse-grained fraction of soil KA. This fraction was previously obtained by means of wet sieving of soil KA that led to significant loss of HS in the sieving residual and thus to a substantial decrease in the amplitude of the spectral signal of OR.

The nature of OR in soils is mostly defined by semiquinones and phenoxyls of different kinds and conjugated bonds (Buchachenko and Wasserman, 1973; Paul et al., 2006; Skrzypczak-Bonduelle et al., 2008). The comparison of the ESR spectra of soil ME, HQ and of leonardite (humic acids) shows a sufficient similarity of signals (Fig. 4, left and right). A slight difference of the spectral line of soil OR from the leonardite spectrum was likely due to its partly overlapping with the $E^{\prime}$ signal of the mineral phase. The spin concentrations of leonardite and leonardite with $10 \%$ of HQ were about four times and three times, respectively, higher than the concentration of OR of soil ME, which only had a content of HS of $2.1 \%$. Comparison of the spectral signals of OR and HQ (Fig. 4, right) hints at a strong influence of conjugated bonds in both substances. The conjugation of bonds in HQ evokes substantial broadening of the corresponding spectral line and a decrease in its amplitude. Comparison of these ESR signals indicates that the spectral line of soil OR was similar to the leonardite signal, which was not strongly broadened. However, the leonardite signal should be substantially overlapped with the signal of mineral paramagnetic defects to be similar to the ESR signal of OR. As shown in Figs. 3 (right) and 4 (left), the amplitude of the spectral line of soil OR strongly depended on the content of HS in soil. The investigation of the soil spectra at W-band (Aleksandrova et al., 2011a) revealed a second spectral line that corresponded to OR with a g-value of approximately 2.003 that confirmed the presence of semiquinone-type radicals in coexistence with carbon-centered "aromatic" radicals (Christofordis et al., 2010; Paul et al., 2006).

Effects of moistening of soil samples and of the $\mathrm{pH}$ value of soil moisture on the concentration of OR were not found (Aleksandrova et al., 2011a).

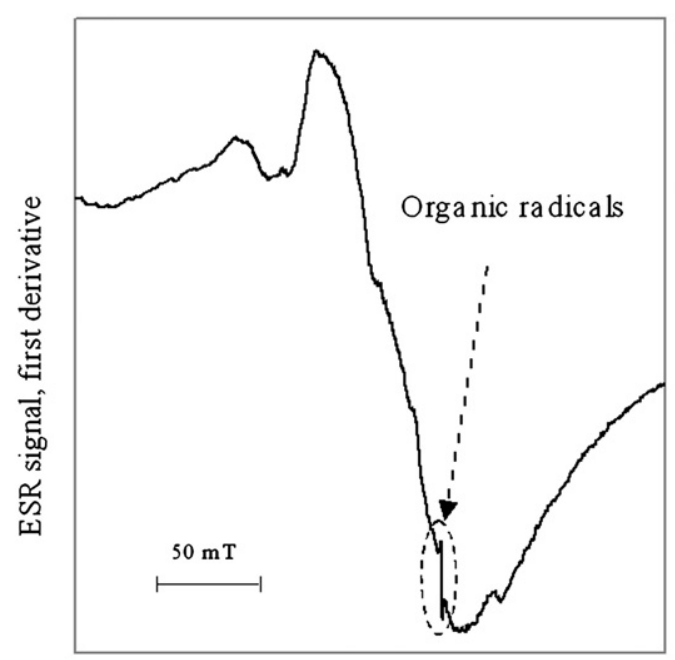

Magnetic field B

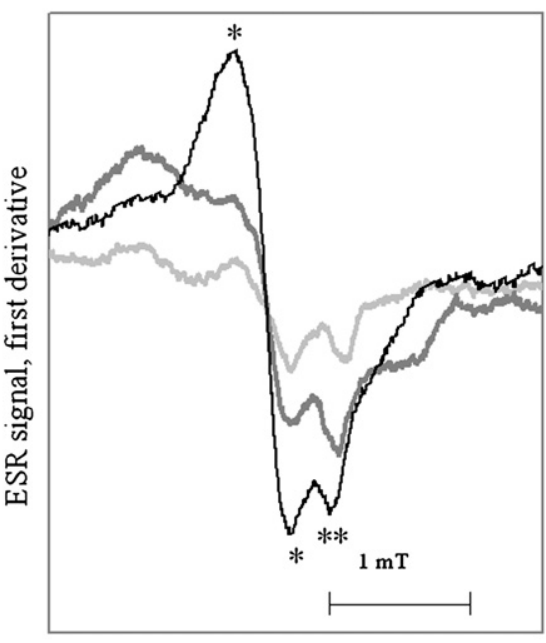

Magnetic field B

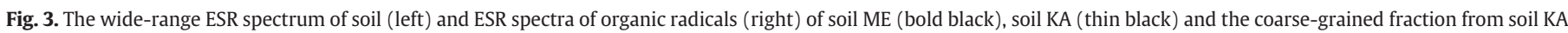
(gray). The main spectral line of OR is shown with asterisk $\left({ }^{*}\right)$. Line of paramagnetic defects is shown with $\left({ }^{* *}\right)$. 

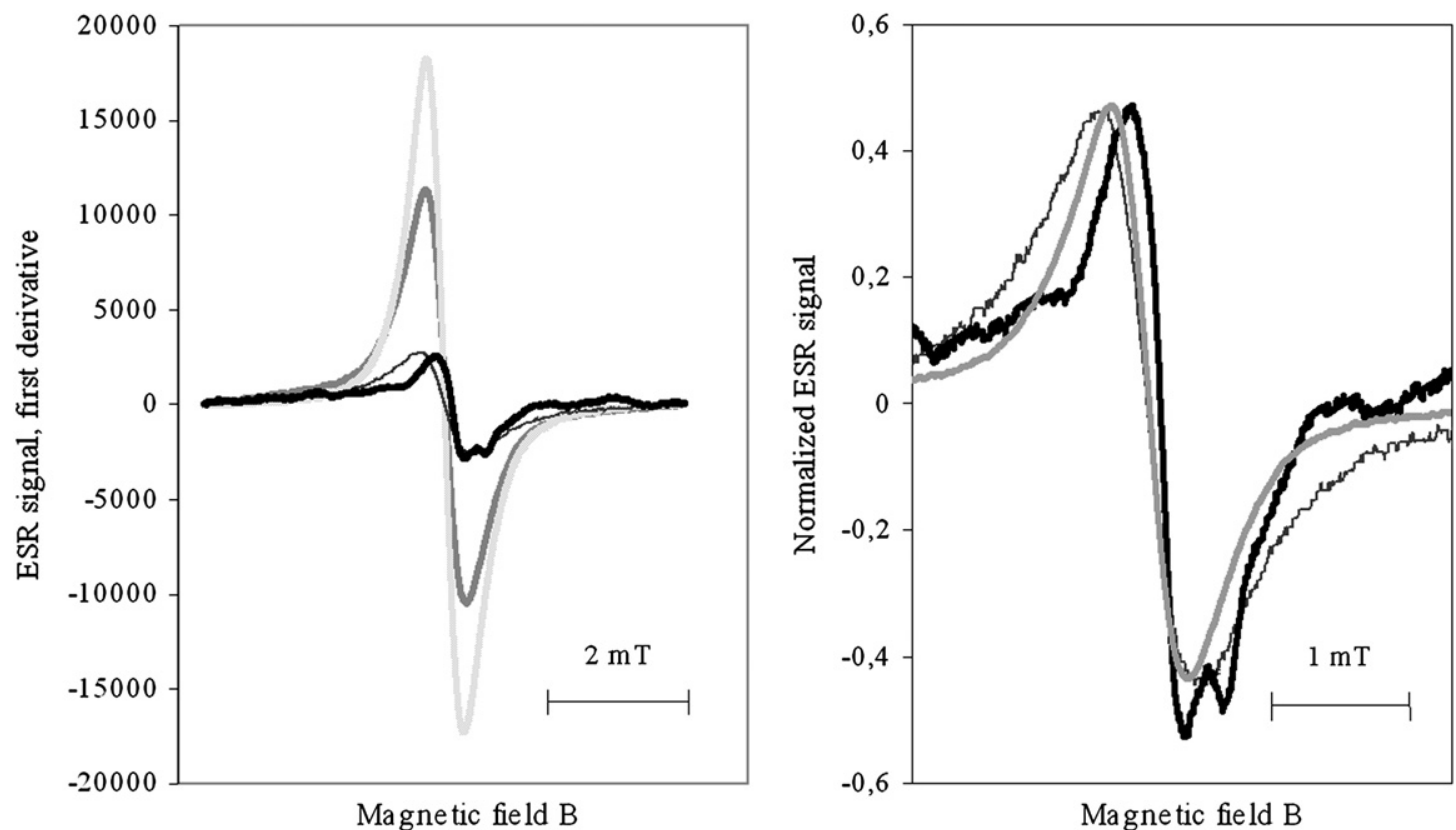

Le onardite $\quad$ Le onardite with HQ (10\%) - Polymerized HQ $\longrightarrow$ s oil ME

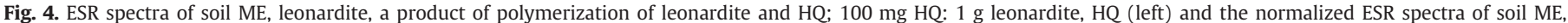
leonardite and HQ (right).

\subsection{Increase in the concentration of soil OR influenced by NR}

The influence of contamination with NR on soil was revealed as a sharp change in the concentration of OR of both soils that in turn was reflected by an increase in the amplitude of their spectral line. The temporal change of the OR spectrum of soil KA labeled with NR 4-Amino Tempo is presented in Fig. 5. Fig. 5 (left) presents the spectra of pure soil KA and of soil KA incubated with 4 Amino Tempo for $3 \mathrm{~min}$ and $6 \mathrm{~min}$, respectively. The spectrum measured after $6 \mathrm{~min}$ exhibits a sharp peak of soil OR. This peak of soil OR disappeared
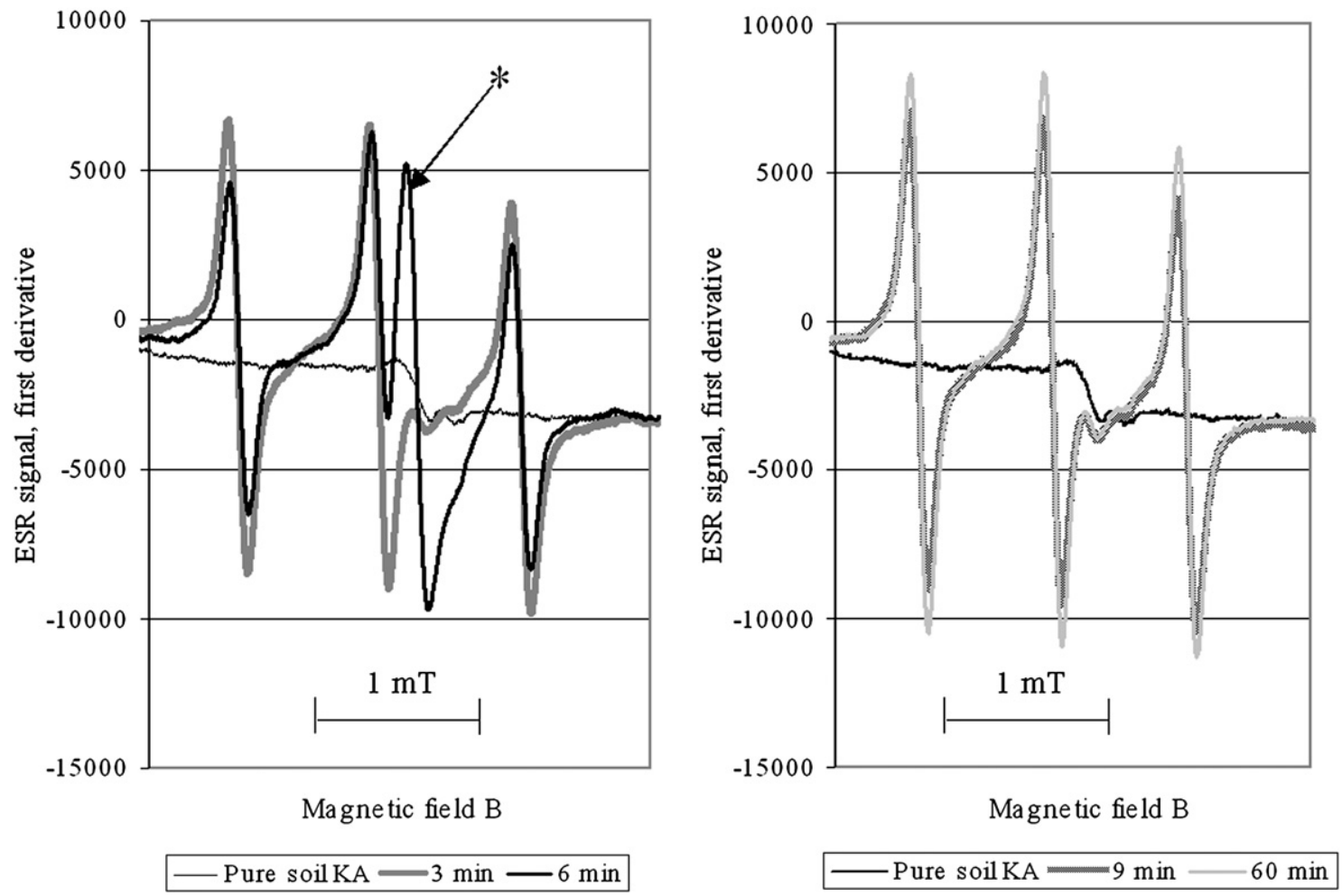

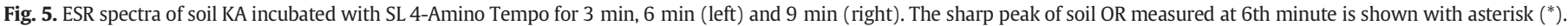


after 9 min of incubation and did not reappear (Fig. 5, right). An appearance of the same peak of soil OR in samples of soil ME was observed after 1-2 days for some days.

An increase in the concentration of HS in the sample resulted in a decrease of the life-time of SL in soil. So, the life-time of 4-Amino Tempo amounted to $4 \mathrm{~min}$ in the sample with leonardite, 12 days in soil ME, and 15 days in soil KA.

Comparison of temporal changes in the ESR spectrum of soil OR with temporal changes in the ESR signal of HQ incubated with radicals of 4-Amino Tempo (Fig. 6, left) indicates that interaction of both, HQ and HS with NR can be modeled as a Teuber reaction (Teuber and Glosauer, 1965). Also temporal changes in the ESR spectrum of NR provide evidence for simultaneous reception of electron and proton from HQ and HS. Otherwise, the spectrum of protonated NR Tempo would have been observed (Khramtsov and Volodarsky, 1998). Therefore, the reduction of the spin concentration of NR seems to be induced by proton and electron transfers from HS to NR.

During ESR experiments, an increase in the amplitude of spectral lines of NR 4-Amino Tempo was followed by their decrease, and subsequently, the same two-way changes in spectral lines were repeated. Thus during the experiment, the concentration of NR differently changed. As shown in Fig. 5 (left), changes in the concentration of NR and OR of soil are tightly connected. A sevenfold increase in the concentration of OR of labeled soil relatively to pure soil is accompanied by a sevenfold decrease in the concentration of NR calculated according to the peak-to-peak method.

An increase in the concentration of OR of soils influenced by 4-Hydroxy Tempo (Fig. 7) and hydrophobic SL Tempo was also observed in experiments with soils (Fig. 8).

Calculation of temporal changes in the OR concentration always showed its significant decrease after several days of interaction. So in Fig. 6 (right), the amplitude of the spectral line of OR of soil KA incubated with SL 4-Amino Tempo for 5 and 12 days was reduced by $20 \%$ and $40 \%$, respectively, relatively to the amplitude of spectral signal of pure soil, while line width was kept constant. Thus, the processes in contaminated soils resulted in a reduction of the initial OR concentration of contaminated soil by as much as $40 \%$.

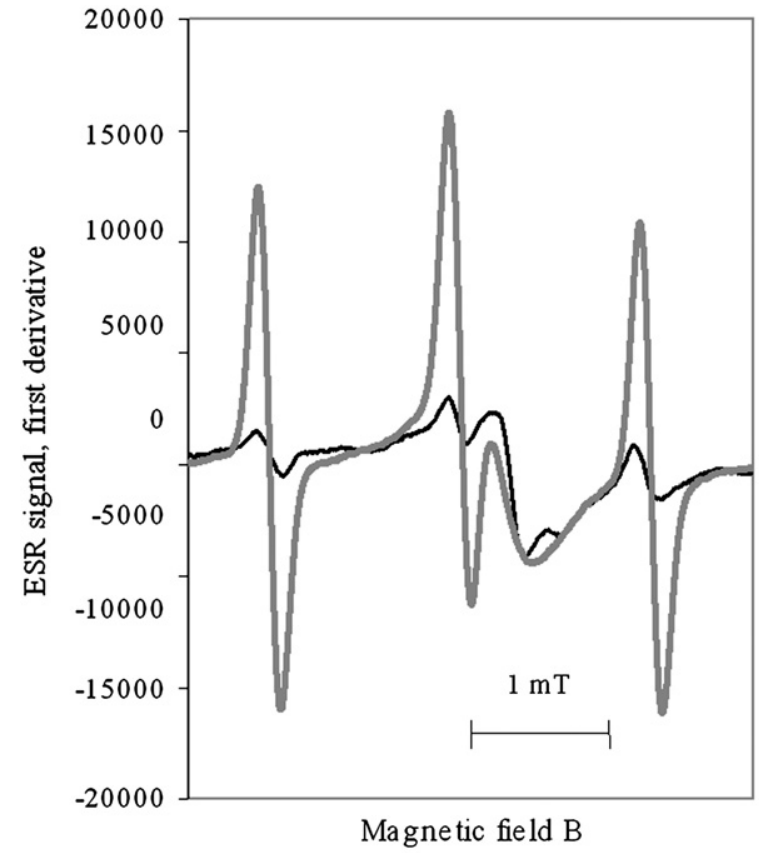

- ME \& Amino - HQ \& Amino
3.3. Investigation of binding of organic xenobiotics to HS via specific functionality and changes in the molecule environment of soil contaminated via labeling

A process of binding of NR to soil HS via specific functional group results in a broadening of their spectral lines (Fig. 6, right). The formation of ESR sub-spectrum of bound NR is detected, beginning with the third day of their incubation with KA. The ESR spectrum of NR 4-Amino Tempo, corresponded to 12 days of their incubation with KA, is slightly overlapped with the sub-spectrum of their bound part in respect to their ESR spectrum, corresponded to 5 days of incubation. As for the interaction between 4-Amino Tempo and soil ME, this process was accompanied by a change in the polarity of the soil molecular environment surrounding nitroxide radicals (Fig. 7).

The hyperfine spectrum of NR is very sensitive to the polarity of the molecular environment. In the ESR experiment, a change in the polarity in the vicinity of nitroxide molecules was detected as splitting of outer spectral lines of NR and their shift towards the central spectral line. NR label and probe the soil molecular environment, influence on it and cause its change, thus characterizing the processes of their interactions with soil.

The temporal change in the hyperfine spectrum of both SL, i.e. 4-Hydroxy Tempo and 4-Amino Tempo, incubated with soil ME pointed at the splitting of ESR spectra and the shift of outer spectral lines towards the central line (Figs. 7, 9, and 10). Therefore in soil ME influenced by the considered NR, the polarity of the environment surrounding NR became less. In soil KA, the spectral splitting was observed only during interaction of soil with 4-Hydroxy Tempo. The ESR spectrum of hydrophobic NR Tempo remained non-split during experiments with both soils (Fig. 8).

The split spectrum of NR 4-Amino Tempo was compared with its ESR spectra in different solvents, such as acetone, ethanol, and hexane (Fig. 10). This comparison between the polarities of NR 4-Amino Tempo in water to the same radicals in acetone allowed for estimating a change in the polarity in the vicinity around NR in soil ME.

The spectral splitting and a shift of spectral lines of NR were accompanied by different peculiarities of changed spectra. In soil KA, the

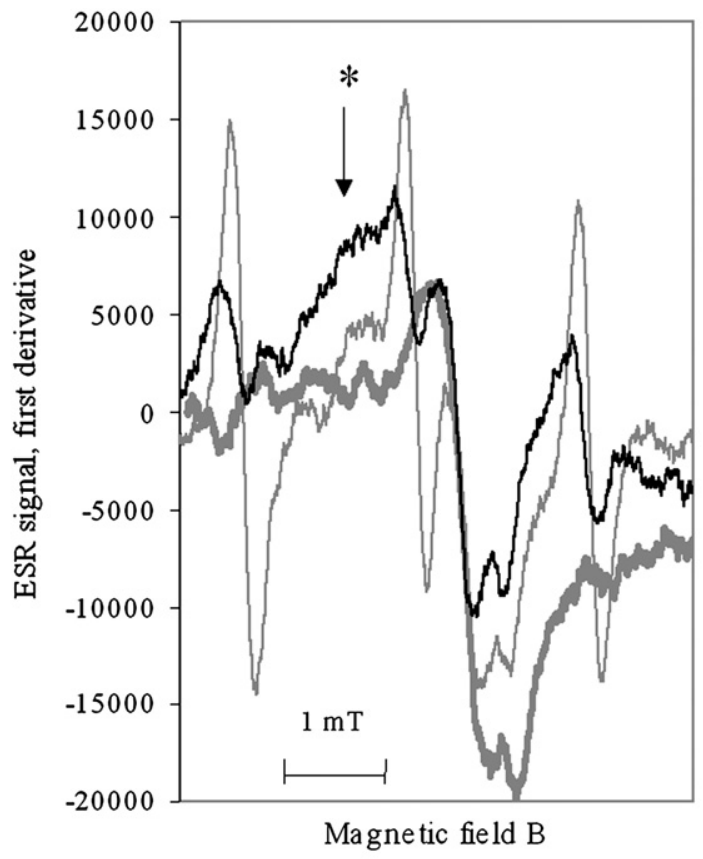

- Pure KA $-5 \mathrm{~d}:-12 \mathrm{~d}$ : KA \& Amino

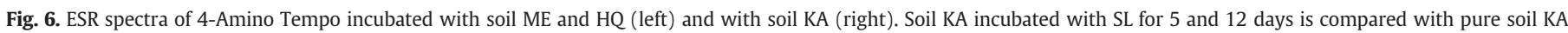
(right). The central line of ESR spectrum of bound nitroxide radicals to soil constituents is shown with asterisk ( $\left.{ }^{*}\right)$ (right). 


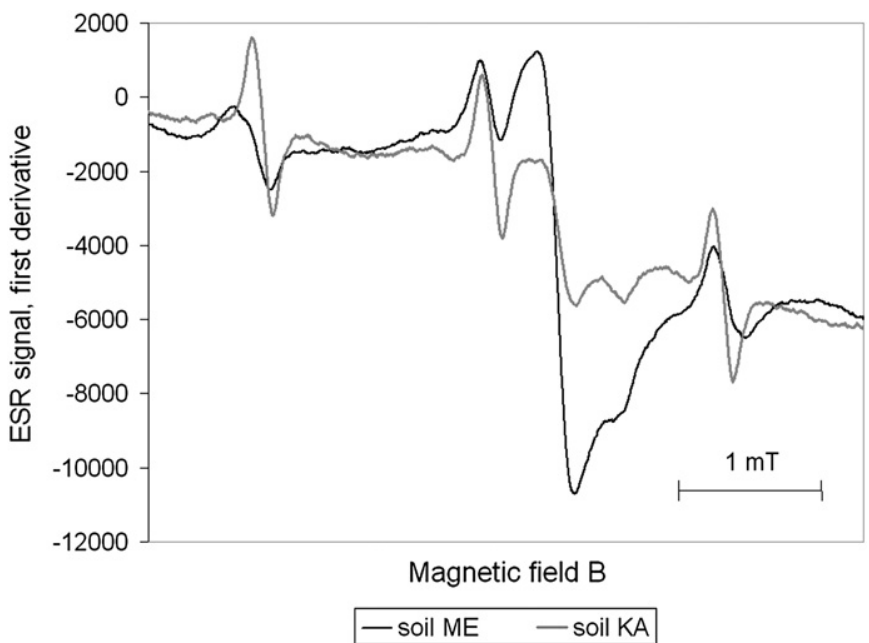

Fig. 7. ESR spectra of SL 4-Hydroxy Tempo incubated with soil KA (gray line) and soil ME (fat black line) for 1 day.

spectral splitting began after some hours of their incubation with soil and was completely finished after 1 day. A shifted spectrum was observed for some days. In soil ME, the formation of a shifted spectrum occurred not as fast as in soil KA. Once established, the shifted spectrum disappeared, because the concentration of SL completely decreased. A shift of the outside spectral lines towards the central line was combined with a slight decrease in line width of NR 4-Hydroxy Tempo, whereas in the case of NR 4-Amino Tempo, the shifted lines were broadened (Figs. 9 and 10). Additional experiments indicated a process of reversible binding of NR 4-Amino Tempo to soil constituents. The comparison with Tempo showed that hydrophobic SL form reversibly bound compounds without any change in the polarity of the molecular environment. Temporal spectra of Tempo indicated a potential process of binding (Fig. 8).

\section{Discussions}

This manuscript presents a new technique developed to investigate the soil molecular environment influenced by organic chemical contaminants with specific functionality. For an application of this technique, soil was labeled with NR, such as 4-Amino Tempo and 4-Hydroxy Tempo, to model contaminants and to measure the changes in the ESR spectra of NR and OR in labeled soil by means of
ESR spectroscopy. Results of this study were compared with results received in the experiment with NR Tempo (Aleksandrova et al., 2011a).

Similar changes in the ESR spectra of OR of soils and HQ influenced by NR likely show that the interaction of contaminants with soil and HQ begins with an exchange of proton and electron pairs (Buchachenko and Wasserman, 1973; Teuber and Glosauer, 1965). Thus, soils exhibit their reactivity by interaction with organic xenobiotic chemicals via formation and transfer of proton and electron pairs to contaminants (Figs. 5 and 6). Only protonation of NR Tempo that would result in an increase of the hyperfine interaction constant (Khramtsov and Volodarsky, 1998) was not observed (Fig. 8). The ESR analyses of the interaction of soil with 4-Hydroxy Tempo and 4-Amino Tempo revealed a decrease in the hyperfine interaction constant. During the experiment, outside spectral lines of NR were only shifted towards the central line (Figs. 7 and 9). The formation of proton and electron pairs accompanied by an appearance of free OR in HS can be considered using a molecular orbital approach (Buchachenko and Wasserman, 1973).

The experimental results showed that NR can return proton and electron pairs (Fig. 5). This process is detected as an initial increase and a subsequent decrease in the concentration of OR of soil originated from semiquinones and phenoxyls of HS. The two-way transfer of pairs seems to be connected with changes in electrochemical properties of the molecular environment of soil influenced by NR, followed by their partial disintegration.

ESR analysis showed that the concentration of NR rapidly reduced in soils enriched with HS. So, life-time of considered NR incubated with $\mathrm{HQ}$, soil ME, soil KA, and a coarse-grained fraction from KA increased in accordance with a decrease in the concentrations of HS. Many researchers pointed at a good resistance of more humified soils against an increased level of toxification (Orlov et al., 2005).

Comparison of the calculated parameters characterizing the interaction of soils KA and ME with NR points at an earlier beginning of transfer the proton's electron pairs by soil KA to NR, whereas reduction of the concentration of NR was faster in soil ME than in soil KA. This peculiarity of soil KA is likely to be connected with a high concentration of iron and a specific texture and requires further investigation. As for Teuber reactions, the oxidation of HQ by NR is sufficiently activated by a reagent, such as $\mathrm{Fe}^{3+}$ (Teuber and Glosauer, 1965). An increased content of iron in soil KA seems to cause an earlier beginning of the interaction between NR and soil HS.

Spectral splitting (Figs. 9 and 10) found for NR 4-Hydroxy Tempo and 4-Amino Tempo indicates that the resulting spectrum contains two spectra, an initial and a shifted one. The temporal change in

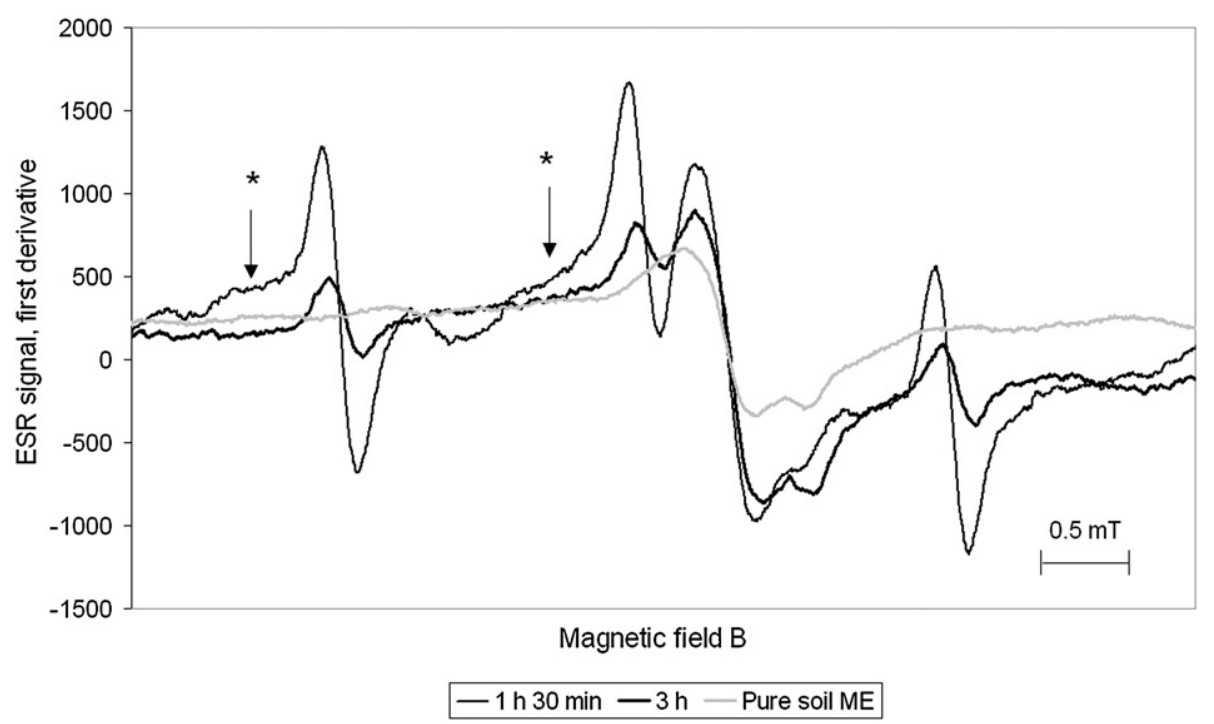

Fig. 8. ESR spectra of SL Tempo incubated with soil ME for 1 h 30 min and 3 h, and of pure soil ME. Asterisks show a sub-spectrum of NR with broadened spectral lines. 


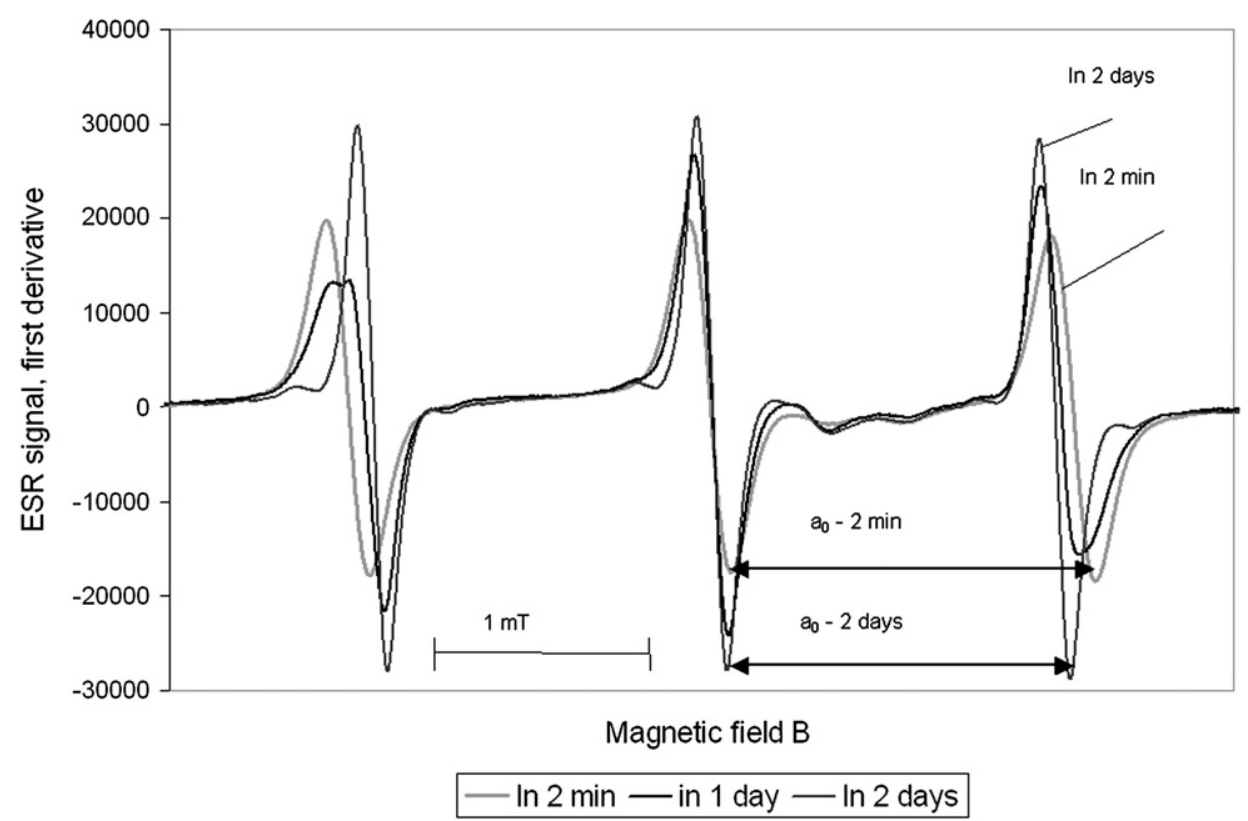

Fig. 9. Split ESR spectrum of soil KA incubated with NR 4-Hydroxy Tempo in comparison with their initial and shifted spectra.

spectrum of NR 4-Hydroxy Tempo incubated with both soils (Fig. 9) shows that one part of NR remains in the initial environment while another part of NR is allocated to a changed environment. Comparison of dynamics of splitting indicates a considerable difference between soils ME and KA. In Fig. 7, the ESR spectrum of NR 4-Hydroxy Tempo incubated with soil ME was still split, and in soil ME, radicals are allocated to the vicinities with different polarities. In the same time, all molecules of NR incubated with soil KA were already allocated to an environment with low polarity. Again, this phenomenon highlights that a change in the polarity of the molecular environment of soil KA began earlier than in soil ME.

The shifted ESR spectrum of NR with a change in line width, hints at an increase in the mobility of radicals of 4-Hydroxy Tempo, whereas radicals of 4-Amino Tempo became less mobile and restricted in their mobility (Figs. 9 and 10). Recent experiments showed unstable, i.e. reversible, binding of NR 4-Amino Tempo to soil ME via amino functionality.

An effect of the specific functionality of contaminants was found. Both considered functionalities caused an increase in the concentration of soil OR and a decrease in the polarity of the molecular environment, as well as different interactions with soil constituents. Restricted mobility of 4-Amino Tempo hints at potential binding of NR to soil constituents via amino functionality.

The experimental results highlighted a new approach to investigate the molecular environment of contaminated soil and a potential process of binding of contaminants to soil constituents via specific functionalities, using labeling of soil with NR. Until present, this method has been widely used in biology, but for the first time, it
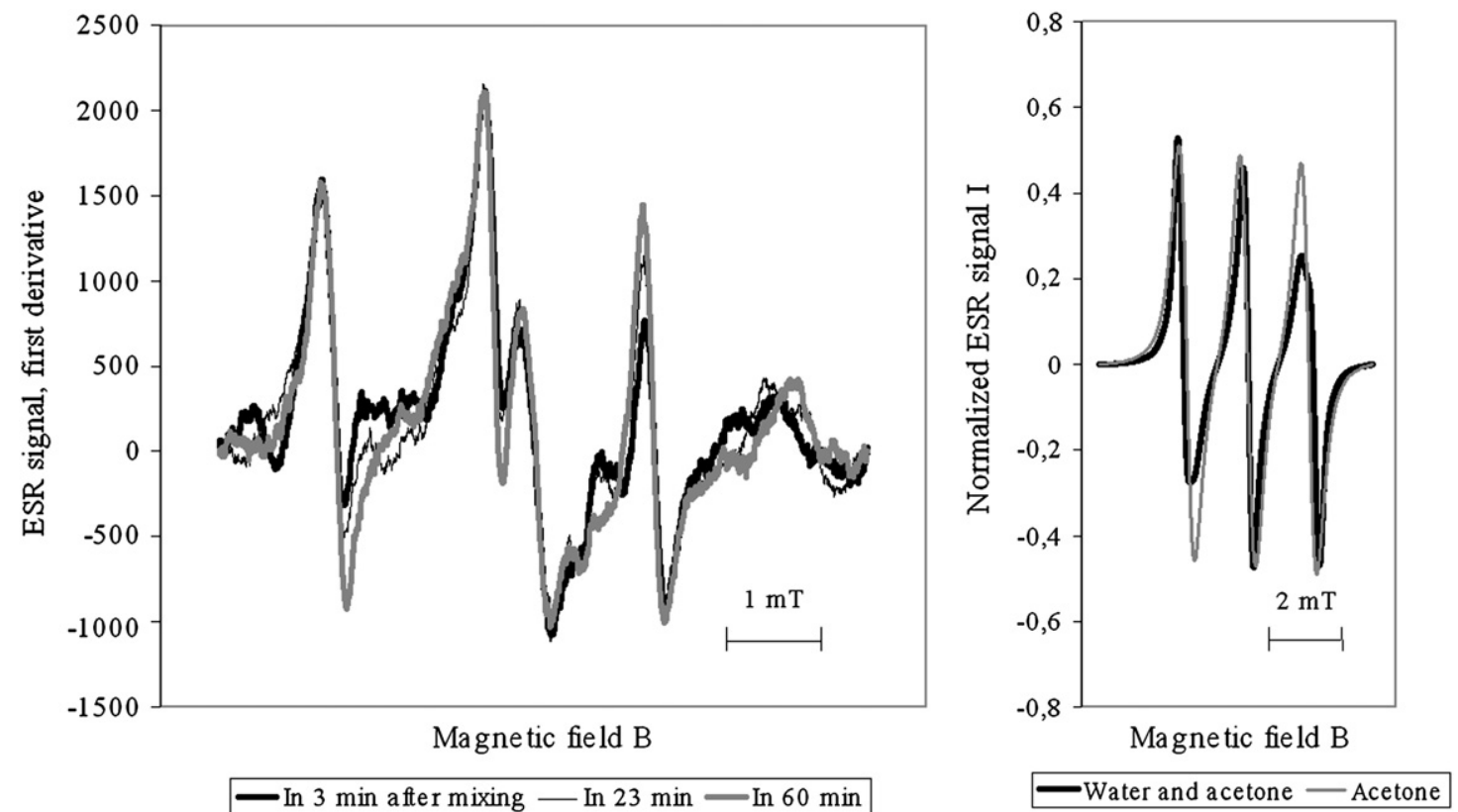

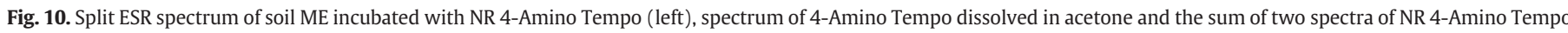
dissolved separately in water and acetone but measured simultaneously (right). 
was applied to soil. An advanced technique promotes a new insight into HS as a quantum system, which response on the external influence, such as moistening or contamination, is substantially defined by the quantum state of HS as a grand canonical Gibbs ensemble and their bonds (Aleksandrova et al., 2011b).

\section{Conclusions}

A new technique of spin labeling is presented that can be applied to soils to characterize the influence of contaminants on changes in the molecular soil environment and their binding to soil constituents via specific functionalities. SL models the soil contaminant and, at the same time, probes the molecular environment surrounding soil molecules and molecules of SL. An advanced technique was applied, which revealed a significant increase in OR of both investigated soils. Based on the experimental results, proton and electron transfer to contaminants supplied by HS in the aqueous solution was hypothesized. This mechanism promotes a decrease in the concentration of NR followed by their subsequent disintegration. Both amino and hydroxy functionalities cause similar decrease in the polarity of the soil molecular environment, but mobility of spin labels changed in different ways. A decrease in the mobility of contaminant molecules SL 4-Amino Tempo hints at a potential binding process of contaminants to soil constituents via amino functional groups. Temporary binding of hydrophobic SL Tempo was detected without a change in the polarity of the molecular environment of soil.

\section{Acknowledgements}

All experiments were carried out at the laboratory of Prof. Dr. HeinzJuergen Steinhoff (Physical Department, University of Osnabrueck, Germany).

I gratefully acknowledge fruitful scientific collaboration with Prof. Dr. Michael Matthies and Prof. Dr. Heinz-Juergen Steinhoff of University Osnabrueck, Germany.

I thank Prof. Dr. Michael Matthies and Prof. Dr. Heinz-Juergen Steinhoff for many enlightening discussions.

I wish to thank Prof. Dr. Irina Perminova of Moscow State University for useful discussions.

\section{References}

Aleksandrova, O.N., Schulz, M., Matthies, M., 2008. Estimation of the influence of humic acid on radionuclide binding to solid residuals in deposits of radioactive waste. Water, Air, and Soil Pollution 194, 287-299.

Aleksandrova, O.N., Schulz, M., Matthies, M., 2010. Natural remediation of surface water systems contaminated with nuclear waste via humic substances in South Ural. Water, Air, and Soil Pollution 206, 203-214.

Aleksandrova, O.N., Steinhoff, H.J., Klasmeier, J., Matthies, M., 2011a. ESR Investigation of Stable Organic Radicals in Soils. Magnetic resonance conference EUROMAR2011 Frankfurt/M, p. 235.

Aleksandrova, O.N., Schulz, M., Matthies, M., 2011b. A quantum statistical approach to remediation effect of humic substances. Water, Air, and Soil Pollution 221, 203-214.

Berliner, L.J., 1979. Spin labelling. Theory and application. Izdatelstvo Mir, Moscow (in Russian).

Berliner, L.J., 1998. Spin labelling. The Next Millennium, 14. Plenum Press, New York.

Bolkova, A.N., 2007. Synthesis and Redox Properties of Humic Substances, Rich in Quinines. Diplom Work, Moscow.

Bollag, Myers, 1992. Detoxification of aquatic and terrestrial sites through binding of pollutants to humic substances. Science of the Total Environment 117-118, 357-366.

Buchachenko, A.L., Wasserman, A.M., 1973. Stable radicals. Electron Shell Structure, its Reactivity and Application.Izdatelstvo Chimia, Moscow (408 pp. (in Russian)).

Chien, Y.-Y., Kim, E.-G., Bleam, W.F., 1997. Paramagnetic relaxation of atrazine solubilized by humic micellar solutions. Environmental Science \& Technology 31, 3204-3208.

Christofordis, K.C., Un, S., Deligiannakis, Y., 2010. Effect of metal ions on the indigenous radicals of humic acids: high field electron paramagnetic resonance study. Environmental Science \& Technology 44, 7011-7016.
Cruz, A.L.N., Gehling, W., Lomnicki, S., Cook, R., Dellinger, B., 2011. Detection of environmentally persistent free radicals at a Superfund Wood treating site. Environmental Science \& Technology 45, 6356-6365.

Eckart, B., 2001. Recovery of uranium- and heavy metal-compounds from radioactive ground water by bonding with pre-treated activated carbon in an underground barrier. Invention Index: DE19925601C02F/001/28

Ferenc, S., 1994. Method for decontamination of radioactive components with ferro/ ferri-cyanid wastes and product for the realization of the method. Inventor Index: HU9403133G21F009/18; C09K017/00. Registration Nr. 1994HU-0003133 19941101.

Ferreira, J.A., Nascimento, O.R., Martin-Neto, L., 2001. Hydrophobic interaction between spin-label 5-SASL and humic acid as revealed by ESR spectroscopy. Environmental Science \& Technology 35, 761-765.

Förster, M., Laabs, V., Lamshoeft, M., Groeneweg, J., Zuehlke, S., Spiteller, M., Krauss, M., Kaupenjohann, M., Amelung, W., 2009. Sequestration of manure-applied sulfadiazine residues in soils. Environmental Science \& Technology 43, 1824-1830.

Fuchs, J., Mehlhorn, R.J., Packer, L., 1989. Free radical reduction mechanisms in mouse epidermis skin homogenates. The Journal of Investigative Dermatology 93 (5), 633-640.

Gulkowska, A., Krauss, M., Rentsch, D., Hollender, J., 2012. Reactions of a sulfonamide antimicribial with model humic constituents: assessing pathways and stability of covalent bonding. Environmental Science \& Technology 46, 2102-2111.

Junge, T., Meyer, K.C., Ciecieleski, K., Adams, A., Schäffer, A., Schmidt, B., 2011. Characterization of non-extractable ${ }^{14} \mathrm{C}$ and ${ }^{13} \mathrm{C}$-sulfadiazine residues in soil including simultaneous amendment of pig manure. Journal of Environmental Science and Health Part B 46, 137-149.

Khramtsov, V.V., Volodarsky, L.B., 1998. Use of imidazoline nitroxides in studies of chemical reactions: ESR measurements of the concentration and reactivity of protons, thiols, and nitric oxide. In: Berliner, L.J. (Ed.), Biological magnetic resonance. Spin Labeling: The Next Millennium, 14. Plenum Press, New York, pp. 109-180.

Kuznetsov, A.N., 1976. The Method of Spin Probing: Principles and Application. Nauka, Moscow (in Russian).

Likhtenshtein, G.I., 1976. Spin Labeling Methods in Molecular Biology. John Wiley \& Sons, Inc., Canada.

Lucarini, M., Mezzina, E., 2011. EPR investigations of organic non-covalent assemblies with spin labels and spin probes. In: Gilbert, et al. (Ed.), Electron Paramagnetic Resonance. The Royal Society of Chemistry, UK, pp. 41-70.

Matthies, M., Witt, J., Klasmeier, J., 2008. Determination of soil biodegradation halflives from simulation testing under aerobic conditions - a kinetic model approach. Environmental Pollution 156, 99-105.

Nowak, K.M., Miltner, A., Gehre, M., Schaeffer, A., Kaestner, M., 2011. Formation and fate of bound residues from microbial biomass during 2,4-D degradation in soil. Environmental Science \& Technology 45, 999-1006.

Orlov, D.S., Sadovnikova, L.K., Suchanova, N.I., 2005. Chemistry of Soils. Izdatelstvo Visshaya shkola, Moscow (558 pp.).

Paul, A., Stoesser, R., Zehl, A., Zwirmann, E., Vogt, R.D., Steinberg, C.E.W., 2006. Nature and abundance of organic radicals in natural organic matter: effect of $\mathrm{pH}$ and irradiation. Environmental Science \& Technology 40, 5897-5903.

Perminova, I.V., Hartfield, K., 2005. Remediation chemistry of humic substances: theory and implications for technology. In: Perminova, I.V., et al. (Ed.), Use of Humic Substances to Remediate Polluted Environments: From Theory to Practice. Springer, The Netherlands, pp. 3-36.

Perminova, I.V., Kulikova, N.A., Zhilin, D.M., Grechishcheva, N.Yu, Kholodov, V.A., Lebedeva, G.F., Matorin, D.N., Venediktov, P.S., Petrosyan, V.S., 2006. Mediating effects of humic substances in aquatic and soil environments. In: Twardowska, I., et al. (Ed.), Viable Methods of Soil and Water Pollution Monitoring, Protection and Remediation. Springer, The Netherlands, pp. 249-273.

Schauss, K., Focks, A., Heuer, H., Kotzerke, A., Schmitt, H., Thiele-Bruhn, S., Wilke, B.-M., Matthies, M., Amelung, W., Klasmeier, J., Scholter, M., 2009. Analysis, fate and effects of the antibiotic sulfadiazine in soil ecosystems. Trends in Analytical Chemistry 28 (5), 612-618

Senesi, N., 1992. Binding mechanisms of pesticides to soil humic substances. The Science of Total Environment 123/124, 63-76.

Skrzypczak-Bonduelle, A, Binet, L, Delpoux, O, Vezin, $\mathrm{H}$, Derenne, S., Robert, F., Gourier, D., 2008. EPR of radicals in primitive organic matter: a tool for the search of biosignatures of the most ancient traces of life. Applied Magnetic Resonance 33, 371-397.

Spagnuolo, M., Jacobson, A.R., Baveye, P., 2005. Electron paramagnetic resonance analysis of the distribution of a hydrophobic spin probe in suspensions of humic acids, hectorite, and aluminium hydroxide-humate-hectorite complexes. Environmental Toxicology and Chemistry 24 (9), 2435-2444.

Stoll, S., 2011. High-field EPR of bioorganic radicals. In: Gilbert, et al. (Ed.), Electron Paramagnetic Resonance. The Royal Society of Chemistry, UK, pp. 107-154.

Teuber, H.-J., Glosauer, O., 1965. Reaktionen mit Nitrosodisulfonat, XXVII ueber die Oxydation einiger weiterer Phenole. Chemische Berichte 98, 2643-2647.

Tikhonov, A.N., 1998. Spin labels. Sorous Educational Journal Biology 1, 8-15 (in Russian).

Voinov, M.A., Smirnov, A.I., 2011. Spin labels and spin probes for measurements of local $\mathrm{pH}$ and electrostatics by EPR. In: Gilbert, et al. (Ed.), Electron Paramagnetic Resonance. The Royal Society of Chemistry, UK, pp. 71-106.

Zarfl, C., Klasmeier, J., Matthies, M., 2009. A conceptual model describing the fate of sulfadiazine and its metabolites observed in manure-amended soils. Chemosphere 77, 720-726. 